\title{
Planificación del desarrollo sostenible: la integración del problema medioambiental en la actividad socioeconómica de la Sierra Norte de Sevilla
}

\author{
Roldán Salgueiro, José L. \\ Ruiz Moreno, Carolina \\ Casillas Bueno, José C. \\ Urbano Mármol, Antonio \\ Río Sánchez, Ángel del \\ Coca Pérez, Agustín \\ González Rodríguez, Ma José \\ Leal Millán, Antonio
}

Departamento de Administración de Empresas y Marketing Universidad de Sevilla

\section{Resumen}

En este trabajo nos hemos propuesto dar a conocer los principios y la problemática del desarrollo sostenible. Un Plan de Desarrollo Sostenible para el Parque Natural de la Sierra Norte de Sevilla es en la actualidad una oportunidad única que se les brinda a las personas e instituciones públicas de reflexionar sobre su futuro, aceptando nuevos objetivos en la interacción de la actividad económica y social con el medio ambiente. En líneas generales, los principios fundamentales que inspiran a un plan de este tipo son: ( I ) satisfacer las necesidades de la generación actual sin comprometer la capacidad para ello de las generaciones futuras, (2) no ocultar los conflictos entre objetivos medioambientales y económicos, sino colocarlos dentro de un marco común de coexistencia y mutuo beneficio y (3) tender no sólo a la creación de riqueza y a la conservación de los recursos naturales y antropológicos, sino también a su justa distribución entre las personas y los territorios.

\section{Palabras clave}

Desarrollo sostenible / Actividad económica / Preservación medioambiental / Equidad social

\section{Introducción}

Actualmente se otorga al tema medioambiental un papel de primer orden en el debate económico. Sin embargo, durante décadas, el argumento fundamental en la economía se había guiado por la idea básica de la expansión de las actividades industriales, olvidando sus posibles repercusiones en la naturaleza.

La gravedad del deterioro ambiental acumulado y sus efectos visibles han conducido a replantear este supuesto y a considerar, tanto desde la teoría económica como desde la esfera política, la variable medioambiental como un factor económico primordial (Muñoz, 1998). Por ello, ha tenido lugar un proceso de integración del medio ambiente en la actividad y el pensamiento económicos hasta llegar a la situación actual, en la que existe un alto nivel de concienciación. Al realizar una revisión de la consideración del medio ambiente en la literatura económica, se pueden distinguir tres etapas claramente diferenciadas (Del Brío y Junquera, 200 I, pp. 21-23):

La primera, que abarca desde comienzos de la industrialización hasta principios de la década de los setenta, se caracteriza por una ausencia de los temas medioambientales en los planteamientos económicos, y se denomina etapa de INOCENCIA MEDIOAMBIENTAL. En ella las empresas fueron pasivas e indiferentes hacia el medio ambiente y se preocupaban exclusivamente de abastecer los mercados para satisfacer la demanda de los consumidores, que para nada consideraban criterios ecológicos.

La publicación del informe del Club de Roma, Los límites del Crecimiento, constituye el hito que marca la transición de la ausencia de los temas ambientales hacia la siguiente fase de preocupación medioambiental, que denominamos etapa del CRECIMIENTO CERO. Se caracteriza por una incipiente atención hacia el medio ambiente en el debate económico. Por otra parte, desde las empresas se comienza a reconocer que sus actividades influyen en el medio ambiente y surgen las primeras iniciativas de protección ambiental en el ámbito empresarial.

En 1987, la publicación del informe Nuestro Futuro común (Informe Brudtland) por la Comisión Mundial sobre el Medio Ambiente y Desarrollo marca un punto de inflexión en la forma de abordar el problema del medio natural hasta nuestros días y constituye el comienzo de la siguiente etapa: el DESARROLLO SOSTENIBLE. En esta fase, las empresas se apoyan en los principios del "desarrollo sostenible" para avanzar en la manera de afrontar los problemas del medio ambiente, tendiendo en las situaciones más activas a la prevención de la contaminación, la minimización de los impactos al medio y de los resi- 


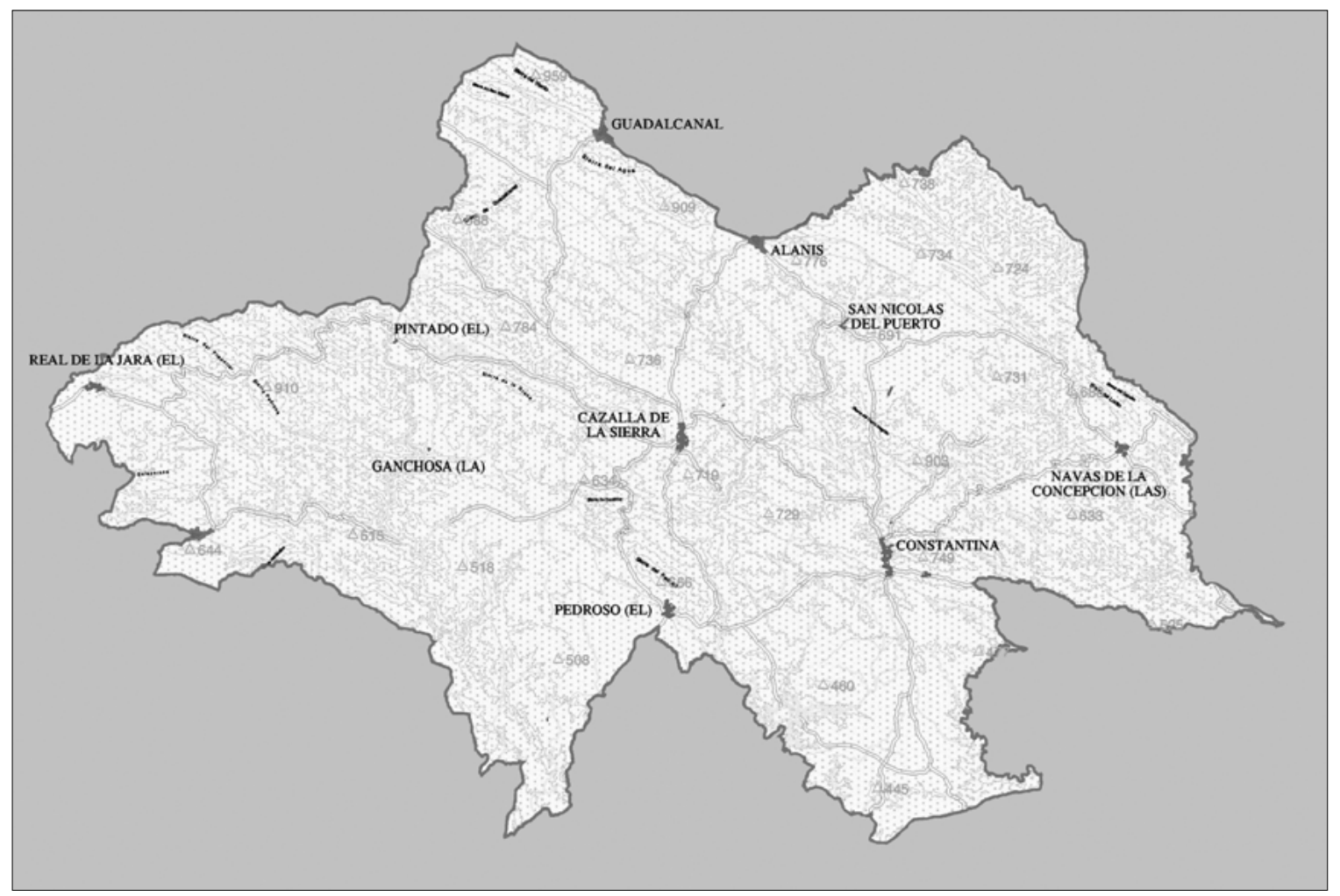

duos generados, la optimización de los consumos y la utilización de tecnologías limpias.

\section{El desarrollo sostenible: qué significa y qué representa}

El desarrollo sostenible supone aceptar un nuevo objetivo en la interacción de la actividad económica y el medio ambiente con el fin de superar los problemas del crecimiento cero. Aunque el término se inscribió por primera vez en un informe de la Unión Internacional para la conservación de la Naturaleza, Estrategia Mundial de Conservación, su uso no se generalizó hasta que la Comisión Mundial del Medio Ambiente y Desarrollo de Naciones Unidas editó su informe bajo el título Nuestro Futuro Común (CMMAD, 1987).

Las ideas más novedosas de ese informe se centraban en los derechos de las generaciones futuras, la prioridad de eliminar la pobreza del momento y la necesidad de encuadrar las acciones humanas dentro de la capacidad de la biosfera. Según el informe de la Comisión, desarrollo sostenible es aquel que satisface las necesidades de la generación presente sin comprometer la capacidad para ello de las generaciones futuras (CMMAD, 1987). En opinión de algunos autores (Gómez, 1991; Jacobs, 1991; Del Brío et Alia, 200I) destacan tres conceptos de su propuesta:

I. El concepto de necesidades. En particular, el informe incorpora un compromiso de equidad global. Esto implica no simplemente la creación de riqueza y la conservación de recursos, sino también su justa distribución.
2. La integración de las consideraciones medioambientales en la toma de decisiones de la política económica, tanto en la teoría como en la práctica. Los conflictos entre los objetivos medioambientales y los económicos no se ocultan, simplemente se los coloca dentro de un marco común en el que pueda reconocerse una variedad de objetivos paralelos.

3. El concepto de sostenibilidad implica la preocupación por la igualdad social entre las generaciones, la cual debe, lógicamente, extenderse a la igualdad social dentro de cada generación.

Las metas fijadas por el informe (desarrollo sostenible, solidaridad intergeneracional y dimensión ambiental de la solidaridad internacional, entre otras) han tenido una aceptación general y han sido admitidas y ratificadas por miles de organizaciones, tanto públicas como privadas, en todo el mundo (Gladwin et alia, 1995).

Sus objetivos fueron reafirmados por los líderes de las naciones industrializadas del Grupo de los 7 en la cumbre de Toronto de 1998, y declarados como un enfoque de política que debía ser aplicado por igual en todo el mundo. Los han adoptado industriales y defensores del medio ambiente, e incluso han entrado a formar parte del lenguaje de los medios de comunicación y del debate académico. Numerosos artículos, libros y conferencias han explicado y definido el término desarrollo sostenible y sus implicaciones.

En estos trabajos se contempla la visión hacia la sostenibilidad como un movimiento alternativo al con- 
flicto entre tecnocentristas y ecocentristas. Desde la postura del desarrollo sostenible, se considera que la naturaleza es el medio en el que vive la humanidad $y$, por tanto, es necesario gestionarla adecuadamente, de forma que se garantice la supervivencia y salud de las poblaciones. Se entiende que el medio ambiente puede absorber y equilibrar las perturbaciones causadas por la actividad económica. Sin embargo, el ecosistema es finito, vulnerable y con una capacidad de regeneración limitada. Esto obliga a cumplir unos axiomas que toman como punto de partida los estudios de las funciones que desempeña la naturaleza en la actividad económica y en la sociedad en general.

El término de funciones naturales fue utilizado por primera vez en el análisis económico por Hueting (1980). Otro autor, De Groot (1993), las definió como la capacidad de los recursos y los procesos naturales para proporcionar productos y servicios que satisfagan las necesidades humanas. Para explicar de forma clara cuáles son las funciones naturales y comprobar qué axiomas se deben cumplir para favorecer los objetivos marcados por el desarrollo sostenible, algunos trabajos han clasificado estas funciones en tres bloques (Ruesga y Durán, 1995; Common, 1998): (1) la provisión de recursos, (2) la absorción de residuos y (3) la provisión de servicios recreativos.

La primera función es la de proporcionar recursos naturales, definidos como cualquier componente del medio ambiente que puede ser utilizado por un organismo, y que, afectando a los procesos de producción y consumo, tienen su origen en fenómenos o procesos naturales que escapan al control del hombre (Romero, 1994).

La segunda función ambiental consiste en asimilar los desechos y residuos generados en las actividades de producción y consumo. El problema que plantea esta función es que sólo cuando la cantidad de los residuos vertidos al medio se encuentran dentro de los límites establecidos por esta capacidad asimilativa, la naturaleza puede mantener su función como depósito.

Finalmente, el medio natural cumple una tercera función, que es la de proporcionar servicios medioambientales. Esta función es menos obvia económicamente que las dos primeras, pero no menos importante (Jacobs, 1991). Hay dos tipos de servicios medioambientales. El primero lo constituyen los atractivos que ofrece el medio ambiente para su consumo directo y consciente. Por ejemplo, brinda espacio para el tiempo libre, así como paisaje y vida silvestre para disfrute estético. Si bien es difícil asignarles valor a estos servicios, es claro que el bienestar humano aumenta considerablemente con ellos.

El segundo tipo de servicio medioambiental puede denominarse, en términos generales, soporte para la vida. Los seres humanos dependen de los procesos naturales que mantienen el funcionamiento de la biosfera. Éstos incluyen funciones tales como el mantenimiento de la diversidad genética (la variedad de especies diferentes), la estabilización de los ecosistemas, el mantenimiento de la composición de la atmósfera y la regulación del clima. Dichos servicios suelen ser intangibles y son consumidos sólo indirectamente (de hecho, la mayoría de las veces inconscientemente), pero no por ello son menos cruciales. Las tres funciones económicas del medio ambiente: provisión de recursos, absorción de residuos y provisión de servicios medioambientales, se pueden ver como componentes de una función general de los ambientes naturales: la relativa al sustento de la vida. Por eso, dada su importancia en la actividad social y económica, y debido a que los daños causados al medio ambiente han puesto en peligro su capacidad para desempeñar estas funciones, desde los planteamientos del desarrollo sostenible se han propuesto varios axiomas, que no afectan directamente al crecimiento económico, pero que sí velan por la conservación del medio ambiente (CMMAD, 1987; Del Brío et Alia, 200 I):

I. Revitalizar el crecimiento, teniendo en cuenta la capacidad de la naturaleza para asimilar los efectos causados por el ser humano.

2. Cambiar la calidad del crecimiento.

3. Satisfacer las necesidades esenciales de trabajo, alimentos, energía, agua e higiene.

4. Asegurar un nivel de población sostenible.

5. Conservar y acrecentar la base de los recursos naturales, empleando antes recursos renovables en vez de no renovables, utilizando estos últimos sólo en la medida que se incremente su disponibilidad y, finalmente, respecto a los primeros, explotarlos sólo en tasas iguales o menores a sus tasas de regeneración.

6. Crear economías sostenibles, es decir, fomentar el uso de energías sostenibles, la industrialización sostenible (que reutilice y recicle los recursos, que minimice los residuos y que preserve el medio ambiente) y la urbanización sostenible.

\section{La naturaleza estratégica de un plan de desarrollo sostenible}

Para ejercer una política territorial se hace necesario un conocimiento profundo de la zona, los agentes sociales, los recursos disponibles, el entorno económico-social y el nivel de satisfacción de los pobladores de ese territorio frente a las acciones que emprenden las Administraciones Públicas. Pero un proceso de planificación del desarrollo requiere además la visión de futuro, el enfoque estratégico o a largo plazo, ese conocimiento de las tendencias y evolución de aquellos componentes del entorno (interno y externo) que plantean amenazas y oportunidades tanto a la población como al territorio $y$, finalmente, las fortalezas y aspectos a potenciar para superar el reto de la supervivencia y el crecimiento. Conviene señalar que la crisis de los setenta sirvió para recordar que los territorios y especialmente ciertas comarcas tienen un comportamiento evoluti- 
vo con ciclos de vida bastantes específicos. Estos ciclos de vida están ligados a los productos y servicios que ofrece, que hacen atractiva su utilización por el hombre.

El proceso, a largo plazo, de la vida y muerte de los territorios y de las ciudades, es imperceptible a corto plazo, en la medida que a factores cuantificables como producto o servicio se suman superestructuras ideológicas y de relación del hombre con el territorio que ha nacido, su lengua y sus formas de comportamiento. Los profundos cambios en la economía actual aceleran el ciclo de vida de los territorios y las ciudades. Al asistir a un cambio de las formas y relaciones de producción se producirán asimismo modificaciones en la superestructura ideológica en las que la estrecha relación actual hombre I territorio nacimiento perderá probablemente su carácter esencial. Ello produce que el futuro de muchos territorios esté en situación crítica y tiene incidencia ya, cuando observamos las diferencias a nivel de tasas de empleo y evolución de la estructura económica de los distintos territorios. Por ello se han puesto en marcha fórmulas que ayuden a gestionar la evolución de los parques naturales y situarlos frente a un futuro aceptable.

Enfrentar futuro a cubrimiento de las necesidades inmediatas no es, en la actualidad una buena discusión política, dada la estrecha relación dialéctica entre las promesas a corto y a largo plazo. Se trata de enfocar las soluciones a corto plazo de manera que sean capaces de adaptarse a las grandes líneas definidas en el largo. Y sólo estas líneas permitirán resolver algunos de los problemas del corto, específicamente los relacionados con el empleo.

Una primera constatación es que el futuro de los territorios no es un problema exclusivo de la Administración, sino que afecta a su población y a los agentes sociales y económicos que actúan en ella.

La Administración, subordinada a las necesidades del territorio, puede impulsar, coordinar y a veces actuar de forma puntual, ayudando a la resolución de los temas más críticos de la vida ciudadana (servicios, infraestructuras, normativa y en algunos casos política industrial o de promoción del empleo). Sin embargo, esta actuación nunca será suficiente para asegurar el proceso constante de reconversión del territorio que resuelva de manera eficiente su futuro y su desarrollo económico y social. Por ello se hace preciso elaborar una estrategia que, por medio de la participación de la administración y la sociedad civil, sirva para el establecimiento de una "cultura de territorio" común y, en el mejor de los casos, situarle en posición competitiva frente al reto que representa el futuro.

Evidentemente, la metodología estratégica va dirigida a anticipar el futuro para competir y adaptarse al entorno para redefinir productos y servicios y maximizar los beneficios de las empresas y de las personas. Situar los territorios al estricto nivel de organi- zaciones que compiten entre sí para acaparar el máximo porcentaje del mercado (en este caso actividades económicas, sociales, culturales y lúdicas) es imposible de entenderlo al pie de la letra en un mundo que ha de tener en cuenta la solidaridad y el desarrollo progresivo de todos sus habitantes.

Sin embargo, frente a una oferta industrial y de servicios que no tenga un valor diferenciador respecto a los restantes territorios, el interés de los pobladores de cada zona es encontrar la especialización en los servicios que se ofrecen, de manera que siga siendo un núcleo de actividades.

Se ha de tener en cuenta que la vida del territorio se define por su nivel de actividad y de transacciones de todo tipo que se realizan en la misma. Los generadores de estas actividades, las empresas, tienen movilidad total en su instalación y sus inversiones van dirigidas a los espacios atractivos por todo tipo de factores: humanos, económicos, de recursos naturales, de servicios, de clima, de comunicaciones, etc.

El proceso actual conlleva, por una parte, la caída de las industrias tradicionales, afectando especialmente a los territorios y ciudades que crecieron durante la primera y segunda industrialización, y por otra, la aparición de nuevos servicios y productos en la constante sustitución de trabajo manual por intelectual. Se genera crisis de empleo, económica y de conversión de recursos humanos. Normalmente, las crisis se abordan, a nivel territorial, intentando plantear una serie de actuaciones puntuales a mayor o menor escala, basadas en la intuición de los gobernantes y principales agentes sociales. Estas propuestas intuitivas sirven para agilizar a corto plazo la situación, pero difícilmente tendrán utilidad para el futuro.

De ahí la necesidad de hacer el paso de integrar intuiciones y análisis de situación y estructurar de manera planificada y participativa las líneas maestras a seguir. Crear "cultura de parque natural" y marcos de referencia para las actividades públicas y privadas ha pasado a ser un objetivo. En muchos territorios las recomendaciones del plan de desarrollo, indicativas para la empresa privada, han pasado a ser imperativos para la Administración Pública, que adecua su programación tradicional al marco que ofrece el plan estratégico.

Aunque es muy difícil definir concretamente un plan de este tipo, las líneas generales que lo guían son en principio las siguientes:

- Es un plan que afecta a todo el territorio y no sólo a su gobierno.

- Está orientado a objetivos a partir de los recursos actuales. De esta orientación sólo selecciona las líneas de propuesta y objetivos considerados como críticos respecto al marco de futuro que se pretende. El resto de múltiples objetivos que puede tener el territorio y sus ciudades se deja seguir con los procedimientos habituales. 
- Tiene en cuenta, explícitamente, el entorno y su evolución, no sólo las necesidades intrínsecas del territorio.

- Analiza a fondo la disponibilidad de recursos entendidos en el sentido mucho más amplio que el estrictamente económico: calificación tecnológica, tradición, situación política, económica, capacidad de generación de recursos (humanos, económicos y tecnológicos), ecología y capacidad de atracción, etc.

- Estudia detalladamente y hace propuestas respecto a los puntos más débiles y sus fuentes de la situación de partida en función de los objetivos críticos.

- Está orientado a la acción controlando con especial énfasis los resultados prácticos.

- Exige la participación activa de todos los agentes sociales y la coordina, basándose en una metodología prefijada.

- Afecta a todo tipo de actividades: económicas, de infraestructura, de zonificación y transportes, enseñanza, etc.

\section{Las interacciones entre el hombre y el entorno: la percepción social del parque natural y el plan de desarrollo sostenible}

El Parque Natural de la Sierra Norte hace referencia a un espacio protegido que ha sido, históricamente, humanizado. Por ello los aspectos "naturales" no pueden ser analizados si no es en relación con el factor humano que ha modelado en gran parte la fisonomía de la Sierra. La ley de Espacios Protegidos (1989) entiende por espacios naturales a proteger las zonas que "no han sido esencialmente modificadas por la acción del hombre, 0 bien lo han sido de tal modo que han generado nuevos ambientes naturales". En el espíritu de la Ley, como vemos, se asemeja el término "espacio natural" a lugares, en cierto modo, inalterados donde todo es producto de la naturaleza, sin apenas verse afectada por la acción antrópica. Este, por supuesto, no ha sido el caso de la Sierra Norte, donde ha existido, históricamente, un volumen importante de hábitat disperso que se demuestra en la numerosa existencia de una arquitectura vernácula diseminada por todo el ámbito serrano y, además, gran parte de la población de los núcleos urbanos trabajaban en la Sierra '. La capacidad de modelar y transformar el paisaje por la acción antrópica es una realidad fácilmente constatable, por lo que el "Parque Natural" ha de entenderse en todas sus dimensiones culturales enfatizando en la complementariedad entre el ser humano y el medio, pues el éxito de conservación de un territorio depende de los vínculos y las interacciones entre el hombre y su entorno.

Así, pues, es necesario a la hora de concebir un plan de desarrollo y de abordar medidas en el Parque Natural, tener en cuenta todo el cúmulo de experiencias y prácticas que se han desarrollado en el seno del territorio: sistemas de cultivos, prácticas ganaderas, actividades de transformación agroindustrial, aprovechamientos mineros, usos forestales, etc. que contribuyen a crear un paisaje cultural que debe ser valorado como una parte más de las señas de identificación de un pueblo.
La declaración de la Sierra Norte como Parque Natural ha seguido criterios ambientalistas, pues las más de 160.000 hectáreas que ocupa, no alberga la totalidad de la Sierra Morena Sevillana, quedando algunas localidades fuera, así como parte de los términos municipales de algunos de los municipios que lo integran. También, los criterios administrativos han delimitado una unidad geológica, geográfica, ecológica y sociocultural en tres espacios continuos: el PN de la Sierra de Aracena y Picos de Aroche y el PN de la Sierra de Hornachuelos, ambos limítrofes.

Esta fragmentación del territorio serrano, contribuye en la ya de por sí acusada desvertebración comarcal. La Ley anteriormente aludida tiene como finalidad: crear infraestructuras y lograr unos niveles de servicios y equipamientos adecuados; mejorar las actividades tradicionales y fomentar otras compatibles con el mantenimiento de los valores ambientales; integrar a los habitantes en las actividades generales por la protección y gestión del PN (art. 13.2) Esto puede originar nuevos conflictos intracomarcales entre las localidades que pugnan por liderar el protagonismo comarcal y aquellas otras que quedan fuera del ámbito de recepción de recursos.

La identificación comarcal en la actualidad se muestra débil, producto de la desvertebración económica a gran escala que se ha producido en la zona en las últimas décadas. Los localismos han brotado o se han acentuado aún más, lo que se demuestra en que apenas existe un asociacionismo de ámbito comarcal por uno más acusado del local. Al mismo tiempo, no existen rituales festivo-ceremoniales que articulen el conjunto de localidades de la Sierra. La pérdida de protagonismo de las ferias de ganado, la ausencia de romerías de proyección comarcal u otros eventos de carácter socioeconómico y cultural hacen dificultoso que se vaya generando una conciencia de pertenencia a un ámbito mayor, y máxime cuando la figura del PN nace fragmentando la propia comarca.

Históricamente se ha producido una fuerte competencia entre las localidades de Constantina y Cazalla de la Sierra por erigirse en cabeceras comarcales. Disputas de tipo económico, administrativo o por la consecución de fondos y recursos externos avivan el conflicto que, como se puede apreciar, está siempre latente en el nivel de los discursos y en las acciones de tipo ritual-simbólico ${ }^{2}$. Por una parte, este conflicto entre estas poblaciones y su hiterland comarcal más inmediato actúa como factor de bloqueo para diseñar estrategias de desarrollo comarcal, pero por otro lado, la pugna por erigirse en cabeceras comarcales activa lazos entre las poblaciones serranas puesto que el ámbito del conflicto no es la localidad sino la comarca. No obstante, en los últimos años ha aparecido en escena la localidad de El Pedroso que, con la ayuda de las instituciones externas está logrando un protagonismo con la celebración, ya en su quinta edición, de la Feria de Muestras de Productos Típicos y Artesanales de la Sierra Norte que, cada año va contando con una nutrida representación de los pueblos de la comarca. 
La percepción que se tiene sobre el territorio varía en gran medida dependiendo del sector social. Si la identidad comarcal es débil producto de la desvertebración económica y social del espacio serrano, la catalogación de parte de este territorio como PN no ha logrado la adhesión de todos los sectores involucrados. Agricultores, ganaderos, comerciantes, industriales, jornaleros, residentes, etc. adoptan distintas posiciones dependiendo de las ventajas o desventajas que la figura del PN afecte a sus intereses. Por poner un ejemplo, los agricultores, desde el inicio mostraron su temor a que se vean frenados la dinámica de despegue económico, ya que se compite con otras zonas en situación de desventaja. No entienden que se limite el pastoreo, o que se impida tocar el monte en zonas de un más del 10\% de pendiente, que lleva a convertir el monte mediterráneo (antropizado) en monte "salvaje", bosques en los que si no se controla el pasto para la ganadería o especies para la caza mayor, los peligros de incendios se incrementan.

Del mismo modo, otros sectores ven limitados o prohibidos algunos aprovechamientos y usos del bosque, tradicionales o modernos, que, en algunos casos, ha supuesto aportes de relativa importancia en las economías domésticas (carboneo, prácticas cinegéticas, recolección de productos diversos, pesca, etc.). Estas restricciones o prohibiciones que son un hecho y que vienen acompañadas de una ausencia de alternativas puede generar en amplias capas de la población una hostilidad a la significación del PN y, no pudiendo dirigirla contra técnicos y administrativos de lo que se entiende como una usurpación de derechos no explicitados, puede volverse en una acción autodestructiva hacia el medio que se pretende proteger.
Es por ello que no se puede conservar mientras que la generalidad de la población inserta en las localidades del PN perciba tal figura como algo ajeno a su vida, o, en el peor de los casos, como cargas u obstáculos para las posibilidades de un hipotético desarrollo futuro.

En definitiva, a través del Plan de Desarrollo Sostenible, se pretende un análisis que ahonde en la relación cambiante de los distintos sectores sociales con el medio, calibrando de forma articulada cuál es la percepción que se tiene sobre el PN y cuál es la que debería adoptarse. Una vez definidos los diversos colectivos socioprofesionales de las localidades de la Sierra Norte, estamos procurando un ejercicio de reflexión colectiva en el que hacerse, entre otras, estas preguntas:

- ¿Quién y por qué se identifica con el PN?

- ¿Es una figura impuesta o se ve como algo propio?

- ¿Es algo positivo para el desarrollo comarcal o un obstáculo?

- ¿Ha supuesto un cambio importante en la relación con el medio?

Todo ello nos llevará a calibrar de mejor modo la percepción "desde dentro" respecto a la visión externa. Es de más provecho para acometer cualquier tipo de actuación conocer el "paisaje de uso" sobre el "paisaje de consumo", reconociendo la legitimidad de la intervención a aquellos que manejan con responsabilidad y perciben el paisaje como legado común, sin que ello suponga negar la legitimidad de la intervención económica, científica y administrativa.

\section{Bibliografía}

CARRERA DÍAZ, G. Carta etnográfica de Constantina. Territorio y paisajes Culturales.

CMMAD. Nuestro futuro común. Madrid: Alianza Editorial, 1987.

COMMON, M. Environmental and Resource Economics: An Introduction. Londres: Logman, 1998.

CONSEJERÍA DE AGRICULTURA Y PESCA. Plan forestal andaluz. Sevilla: Junta de Andalucía, 1990.

DE GROOT, W.T. Environmental Science Theory: Concepts and Methods in a One-World, Problem-Oriented Paradigm. Dordrecht: Elsevier, 1993.

DEL BRIO, ..; JUNQUERA, B. Medio ambiente y empresa. Madrid: Cívitas, 2001.

GLADWIN, T.N.; KENNELLY, J.j; KRAUSE, T.S. Shifting Paradigms for Sustainable Development: Implications for Management
Theory and Research. Academy of Management Review, v. 20, $\mathrm{n}^{\circ}$ 4, 1995, pp. 874-907.

GÓMEZ, J.A. Estrategias para el desarrollo sostenible. Sistema, $\mathrm{n}^{\circ}$ 104-105, 1991, pp. 47-76

HU, GETING, R. (1980): New Scarcity and Economic Growth. Amsterdam: North-Holland, 1980.

JACOBS, M. The Green Economy. Londres: Pluto Press, 199|.

MUÑOZ, C. La Innovación dentro de una estrategia de desarrollo sustentable. Comercio Exterior, diciembre, 1998, pp. 982986.

ROMERO, C. Economía de los recursos ambientales y naturales. Madrid: Alianza Editorial, 1994.

RUESGA, S.M.; DURÁN, G. La empresa y el medio ambiente. Pirámide, Madrid. 1995.

\section{Notas}

I. Por poner el ejemplo de Constantina, en 1900, 2244 personas habitaban en diseminados por 768 I que lo hacían en el casco urbano. En 1950 la proporción es de 2733 por I I 886, en 1970 es de 686 por 10228 para llegar en 1990 a 545 habitantes diseminados por 7335 en el casco (Gema Carrera: 67)
2. Significativo es que no fraguara una denominación de origen para los anisados que llevara los nombres de CazallaConstantina al modo de Montilla-Moriles, siendo el licor en ambas localidades de similares características, aunque en cada una de ellas defiendan y cataloguen el propio como "bastante mejor". 